\title{
Relativity constraints on the two-nucleon contact interaction
}

\author{
L. Girlanda ${ }^{\mathrm{a}, \mathrm{b}}$, S. Pastore ${ }^{\mathrm{c}}$, R. Schiavilla ${ }^{\mathrm{c}, \mathrm{d}}$, and M. Viviani ${ }^{\mathrm{b}}$ \\ ${ }^{a}$ Department of Physics, University of Pisa, 56127 Pisa, Italy \\ ${ }^{\mathrm{b}}$ INFN-Pisa, 56127 Pisa, Italy \\ ${ }^{\mathrm{c}}$ Department of Physics, Old Dominion University, Norfolk, VA 23529, USA \\ d Jefferson Lab, Newport News, VA 23606, USA
}

(Dated: December 7, 2018)

\begin{abstract}
We construct the most general, relativistically invariant, contact Lagrangian at order $Q^{2}$ in the power counting, $Q$ denoting the low momentum scale. A complete, but non-minimal, set of (contact) interaction terms is identified, which upon non-relativistic reduction generate 2 leading independent operator combinations of order $Q^{0}$ and 7 sub-leading ones of order $Q^{2}$-a result derived previously in the heavy-baryon formulation of effective field theories (EFT's). We show that Poincaré covariance of the theory requires that additional terms with fixed coefficients be included, in order to describe the two-nucleon potential in reference frames other than the centerof-mass frame. These terms will contribute in systems with mass number $A>2$, and their impact on EFT calculations of binding energies and scattering observables in these systems should be studied.
\end{abstract}

PACS numbers: 12.39.Fe, 21.30.-x, 11.30.Cp, 13.75.Cs 


\section{INTRODUCTION AND CONCLUSIONS}

Chiral effective field theory ( $\chi$ EFT), pioneered by Weinberg in a series of seminal papers almost two decades ago [1], has led to a novel understanding of strong interactions in nuclei by providing a direct link between these interactions and the symmetries of quantum chromodynamics, including chiral symmetry with its explicit and dynamical breaking mechanisms (see review papers in Refs. [2]). In its original form, $\chi$ EFT is formulated in terms of pions and (non-relativistic) nucleons, whose interactions, strongly constrained by chiral symmetry, are organized as an expansion in powers of small momenta $Q$. All heavier degrees of freedom are "integrated out", and their effects are implicitly subsumed in the coupling constants accompanying local vertices. At sufficiently low energy, even the pions can be integrated out, and the nucleons only interact through contact vertices. In either case, two-nucleon $(N N)$ contact interactions are an important aspect of EFT descriptions. In the present paper we examine the constraints that relativistic covariance imposes on the resulting $N N$ potential up to order $Q^{2}$ (or next-to-next leading order, $\mathrm{N}^{2} \mathrm{LO}$ ).

At LO $\left(Q^{0}\right)$ in the low energy expansion there are only 2 independent contact interactions [1]

$$
\mathcal{L}_{I}^{(0)}=-\frac{1}{2} C_{S} O_{S}-\frac{1}{2} C_{T} O_{T}
$$

where $C_{S}$ and $C_{T}$ denote low-energy constants (LEC's), and the operators $O_{S}$ and $O_{T}$ are defined in terms of the non-relativistic nucleon field $N(x)$,

$$
N(x)=\int \frac{d \mathbf{p}}{(2 \pi)^{3}} b_{s}(\mathbf{p}) \chi_{s} \mathrm{e}^{-i p \cdot x},
$$

and its adjoint in Table I. Here $b_{s}(\mathbf{p})$ and $b_{s}^{\dagger}(\mathbf{p})$ are annihilation and creation operators for a nucleon in spin state $s$, satisfying standard anticommutation relations, i.e. $\left[b_{s}(\mathbf{p}), b_{s^{\prime}}^{\dagger}\left(\mathbf{p}^{\prime}\right)\right]_{+}=(2 \pi)^{3} \delta\left(\mathbf{p}-\mathbf{p}^{\prime}\right) \delta_{s s^{\prime}}$. A sum over the repeated index $s= \pm 1 / 2$ is implied, and it is understood that field operator products are normal-ordered in $O_{S}$ and $O_{T}$ (as well as in the $O_{i}$ 's defined below). We have suppressed isospin indices, since they will not enter in the discussion to follow (see Sec. IIA). The corresponding $N N$ potential reads

$$
v^{\mathrm{CT} 0}=C_{S}+C_{T} \boldsymbol{\sigma}_{1} \cdot \boldsymbol{\sigma}_{2} \text {. }
$$

At the next non-vanishing order, $\mathrm{N}^{2} \mathrm{LO}$, the contact Lagrangian involving two derivatives of the nucleon fields has been written in Ref. [3] as consisting of 14 operators

$$
\mathcal{L}_{I}^{(2)}=-\sum_{i=1}^{14} C_{i}^{\prime} O_{i},
$$

where the $O_{i}$ 's are listed in Table $\llbracket$ and the $C_{i}^{\prime}$ are LEC's. In fact, we showed in Ref. [4] that, after partial integrations, only 12 out of the above 14 operators are independent, since

$$
O_{7}+2 O_{10}=O_{8}+2 O_{11}, \quad O_{4}+O_{5}=O_{6} .
$$

In a general frame in which the $N N$ pair has total momentum $\mathbf{P}$ and initial and final relative momenta, respectively, $\mathbf{p}$ and $\mathbf{p}^{\prime}$, the potential derived from the Lagrangian $\mathcal{L}_{I}^{(2)}$ is 


\begin{tabular}{c|c}
\hline \hline$O_{S}$ & $\left(N^{\dagger} N\right)\left(N^{\dagger} N\right)$ \\
$O_{T}$ & $\left(N^{\dagger} \boldsymbol{\sigma} N\right) \cdot\left(N^{\dagger} \boldsymbol{\sigma} N\right)$ \\
\hline$O_{1}$ & $\left(N^{\dagger} \vec{\nabla} N\right)^{2}+$ h.c. \\
$O_{2}$ & $\left(N^{\dagger} \vec{\nabla} N\right) \cdot\left(N^{\dagger} \overleftarrow{\nabla} N\right)$ \\
$O_{3}$ & $\left(N^{\dagger} N\right)\left(N^{\dagger} \vec{\nabla}^{2} N\right)+$ h.c. \\
$O_{4}$ & $i\left(N^{\dagger} \vec{\nabla} N\right) \cdot\left(N^{\dagger} \overleftarrow{\nabla} \times \boldsymbol{\sigma} N\right)+$ h.c. \\
$O_{5}$ & $i\left(N^{\dagger} N\right)\left(N^{\dagger} \overleftarrow{\nabla} \cdot \boldsymbol{\sigma} \times \vec{\nabla} N\right)$ \\
$O_{6}$ & $i\left(N^{\dagger} \boldsymbol{\sigma} N\right) \cdot\left(N^{\dagger} \overleftarrow{\nabla} \times \vec{\nabla} N\right)$ \\
$O_{7}$ & $\left(N^{\dagger} \boldsymbol{\sigma} \cdot \vec{\nabla} N\right)\left(N^{\dagger} \boldsymbol{\sigma} \cdot \vec{\nabla} N\right)+$ h.c. \\
$O_{8}$ & $\left(N^{\dagger} \sigma^{j} \overrightarrow{\nabla^{k}} N\right)\left(N^{\dagger} \sigma^{k} \overrightarrow{\nabla^{j}} N\right)+$ h.c. \\
$O_{9}$ & $\left(N^{\dagger} \sigma^{j} \vec{\nabla}^{k} N\right)\left(N^{\dagger} \sigma^{j} \overrightarrow{\nabla^{k}} N\right)+$ h.c. \\
$O_{10}$ & $\left(N^{\dagger} \boldsymbol{\sigma} \cdot \vec{\nabla} N\right)\left(N^{\dagger} \overleftarrow{\nabla} \cdot \boldsymbol{\sigma} N\right)$ \\
$O_{11}$ & $\left(N^{\dagger} \sigma^{j} \overrightarrow{\nabla^{k}} N\right)\left(N^{\dagger} \overleftarrow{\nabla^{j}} \sigma^{k} N\right)$ \\
$O_{12}$ & $\left(N^{\dagger} \sigma^{j} \overrightarrow{\nabla^{k}} N\right)\left(N^{\dagger} \overleftarrow{\nabla^{k}} \sigma^{j} N\right)$ \\
$O_{13}$ & $\left(N^{\dagger} \overleftarrow{\nabla} \cdot \boldsymbol{\sigma} \overrightarrow{\nabla^{j}} N\right)\left(N^{\dagger} \sigma^{j} N\right)+$ h.c. \\
$O_{14}$ & $2\left(N^{\dagger} \overleftarrow{\nabla} \sigma^{j} \cdot \vec{\nabla} N\right)\left(N^{\dagger} \sigma^{j} N\right)$ \\
\hline \hline
\end{tabular}

TABLE I: Operators entering the LO $\left(Q^{0}\right)$ and $\mathrm{N}^{2} \mathrm{LO}\left(Q^{2}\right)$ contact interactions [3]. The left (right) arrow on $\nabla$ indicates that the gradient acts on the left (right) field. Normal-ordering of the field operator products is understood.

conveniently separated into a term, $v^{\mathrm{CT} 2}$, independent of $\mathbf{P}[\underline{3}, 5]$ and one, $v_{\mathbf{P}}^{\mathrm{CT} 2}$, dependent on it [4]:

$$
\begin{aligned}
v^{\mathrm{CT} 2}(\mathbf{k}, \mathbf{K})= & C_{1} k^{2}+C_{2} K^{2}+\left(C_{3} k^{2}+C_{4} K^{2}\right) \boldsymbol{\sigma}_{1} \cdot \boldsymbol{\sigma}_{2}+i C_{5} \frac{\boldsymbol{\sigma}_{1}+\boldsymbol{\sigma}_{2}}{2} \cdot \mathbf{K} \times \mathbf{k} \\
+ & C_{6} \boldsymbol{\sigma}_{1} \cdot \mathbf{k} \boldsymbol{\sigma}_{2} \cdot \mathbf{k}+C_{7} \boldsymbol{\sigma}_{1} \cdot \mathbf{K} \boldsymbol{\sigma}_{2} \cdot \mathbf{K}, \\
v_{\mathbf{P}}^{\mathrm{CT} 2}(\mathbf{k}, \mathbf{K}) & =i C_{1}^{*} \frac{\boldsymbol{\sigma}_{1}-\boldsymbol{\sigma}_{2}}{2} \cdot \mathbf{P} \times \mathbf{k}+C_{2}^{*}\left(\boldsymbol{\sigma}_{1} \cdot \mathbf{P} \boldsymbol{\sigma}_{2} \cdot \mathbf{K}-\boldsymbol{\sigma}_{1} \cdot \mathbf{K} \boldsymbol{\sigma}_{2} \cdot \mathbf{P}\right) \\
& +\left(C_{3}^{*}+C_{4}^{*} \boldsymbol{\sigma}_{1} \cdot \boldsymbol{\sigma}_{2}\right) P^{2}+C_{5}^{*} \boldsymbol{\sigma}_{1} \cdot \mathbf{P} \boldsymbol{\sigma}_{2} \cdot \mathbf{P},
\end{aligned}
$$

where the momenta $\mathbf{k}$ and $\mathbf{K}$ are defined as $\mathbf{k}=\mathbf{p}^{\prime}-\mathbf{p}$ and $\mathbf{K}=\left(\mathbf{p}^{\prime}+\mathbf{p}\right) / 2$, and the $C_{i}$ 's $(i=1, \ldots, 7)$ and $C_{i}^{*}(i=1, \ldots, 5)$ are in a one-to-one correspondence with the LEC's $C_{i}^{\prime \prime}$ 's multiplying the 12 independent operators (see Refs. [3 5$]$ ).

We argued in Ref. [4] that the $\mathbf{P}$-dependent terms represent boost corrections to the LO potential $v^{\mathrm{CT} 0}$, and that the $C_{i}^{*}$, rather than being independent LEC's, are in fact related to $C_{S}$ and $C_{T}$ as

$$
C_{1}^{*}=\frac{C_{S}-C_{T}}{4 m^{2}}, \quad C_{2}^{*}=\frac{C_{T}}{2 m^{2}}, \quad C_{3}^{*}=-\frac{C_{S}}{4 m^{2}}, \quad C_{4}^{*}=-\frac{C_{T}}{4 m^{2}}, \quad C_{5}^{*}=0,
$$

where $m$ is the nucleon mass. This result is derived in relativistic quantum mechanics - its instant-form formulation [6] - by requiring that the commutation relations of the Poincaré group generators are satisfied, which, to order $\mathbf{P}^{2} / m^{2}$, leads to the elegant relation [7, 8]

$$
v_{\mathbf{P}}^{\mathrm{CT} 2}=-\frac{P^{2}}{8 m^{2}} v^{\mathrm{CT} 0}+\frac{i}{8 m^{2}}\left[\mathbf{P} \cdot \mathbf{r} \mathbf{P} \cdot \mathbf{p}, v^{\mathrm{CT} 0}\right]+\frac{i}{8 m^{2}}\left[\left(\boldsymbol{\sigma}_{1}-\boldsymbol{\sigma}_{2}\right) \times \mathbf{P} \cdot \mathbf{p}, v^{\mathrm{CT} 0}\right],
$$


where $\mathbf{r}$ and $\mathbf{p}$ are, respectively, the relative position and momentum operators. The potential $v_{\mathbf{P}}^{\mathrm{CT} 2}(\mathbf{k}, \mathbf{K})$ then follows by evaluating the commutators in momentum space, and by retaining only contributions of order $Q^{2}$ (we assume here $P \sim k \sim K \sim Q$ ). That there are dynamical corrections to the $N N$ interaction, when it is boosted to an arbitrary frame, is not surprising, as in instant-form relativistic quantum mechanics interactions enter both the Hamiltonian and boost generators.

In the present paper, we justify the claim made above in a EFT setting. We proceed in two steps. First, we construct, up to order $Q^{2}$ included, the most general hermitian Lagrangian density allowed by invariance under transformations of the Lorentz group and by the discrete symmetries of the strong interaction. After performing its non-relativistic reduction, we find that there are 2 independent operator combinations of order $Q^{0}$, accompanied by specific $Q^{2}$ corrections, and 7 independent operator combinations of order $Q^{2}$ - a result also obtained [9] in the heavy baryon formulation [10] of $\mathcal{L}_{I}^{(2)}$ by requiring that it be re-parametrization invariant [11].

Second, we show that this same picture emerges within the non-relativistic theory in the context of a systematic power counting, by enforcing that the commutation relations among the Poincaré group generators are satisfied order by order in the low energy expansion (for a similar approach, in a different context, see Ref. [12]). The above correspondence between the $C_{i}^{*}$ 's and $C_{S}, C_{T}$ is recovered, showing that the commutator relation in Eq. (1.9) remains valid in a EFT framework. Thus, in order to determine the boost corrections of order $Q^{2}$ to the complete LO chiral potential, which also includes the one-pion-exchange term, one can either use Eq. (1.9) or compute the potential in an arbitrary frame starting from the Lagrangian of the covariant theory.

These boost corrections should be taken into account in $\chi \mathrm{EFT}$ (and EFT) calculations of nuclei with mass number $A>2$. So far, they have been evaluated, for the case of realistic potentials, in the $A=3$ and 4 binding energies, where they have been found to give, respectively, about $400 \mathrm{keV}$ and $1.9 \mathrm{MeV}$ repulsive contributions [8], as well as in threenucleon scattering observables [13], where, in particular, they have led to an increase of the discrepancy between theory and experiment in the $n d$ vector analyzing power.

\section{NON-RELATIVISTIC REDUCTION}

To begin with, we observe that, while the relativistic theory is written in terms of fermion fields $\psi=\psi^{(+)}+\psi^{(-)}$containing both positive- and negative-energy components, the latter play no role in the $N N$ contact potential at order $Q^{2}$ of interest here. This is because antinucleon degrees of freedom only enter via loop corrections, and each loop is suppressed by a factor $Q^{3}$ in time ordered perturbation theory (examples are shown in Fig. 1). Hence, the two-derivative contact Lagrangian (of order $Q^{2}$ ) can be derived, without any loss of generality, starting from the relativistic theory and ignoring the negative energy components.

\section{A. Generalities and strategy}

The building blocks of the relativistic contact Lagrangian are products of fermion bilinears with space-time structures

$$
\frac{1}{(2 m)^{N_{d}}}\left(\bar{\psi} i \overleftrightarrow{\partial}^{\alpha} i \overleftrightarrow{\partial}^{\beta} \cdots \Gamma_{A} \psi\right) \partial^{\lambda} \partial^{\mu} \cdots\left(\bar{\psi} i \overleftrightarrow{\partial}^{\sigma} \overleftrightarrow{i \partial}^{\tau} \cdots \Gamma_{B} \psi\right)
$$



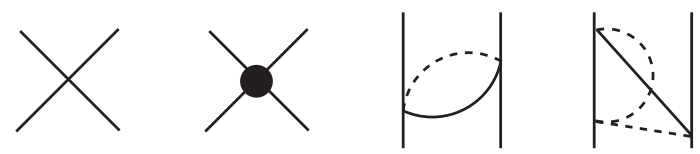

FIG. 1: Time ordered diagrams contributing to the $N N$ scattering amplitude and involving nucleons (solid lines) and antinucleons (dashed lines) interacting through the contact vertices at order $Q^{0}$ and $Q^{2}$ (solid circle). Note that at order $Q^{2}$ the diagrams with antinucleons do not contribute (see text for explanation).

where $\overleftrightarrow{\partial}^{\alpha}=\vec{\partial}^{\alpha}-\overleftarrow{\partial}^{\alpha}$ and the $\Gamma^{\prime}$ s denote generic elements of the Clifford algebra, expanded in the usual basis $1, \gamma_{5}, \gamma^{\mu}, \gamma^{\mu} \gamma_{5}, \sigma^{\mu \nu}$, or the Levi-Civita tensor $\epsilon^{\mu \nu \rho \sigma}$ (with the convention $\left.\epsilon^{0123}=-1\right)$. In the above equation, $N_{d}$ stands for the number of four-gradients (both $\overleftrightarrow{\partial}$ and $\left.\partial^{\lambda}\right)$ entering the formula, and the factor $1 /(2 m)^{N_{d}}$ has been introduced so that all contact terms will have the same dimension. The Lorentz indices $\alpha, \ldots, \tau$ on the partial derivatives are contracted among themselves and/or with those in the $\Gamma_{A, B}$ (for ease of presentation, these indices, unless necessary, will be suppressed hereafter). In order to have flavor singlets, the isospin structure of the two bilinears must be either $1 \otimes 1$ or $\tau^{a} \otimes \tau^{a}$. However, the latter needs not be considered, as it can be eliminated by Fierz rearrangement.

A few remarks are now in order. First, the Lagrangian density should be hermitian and invariant under charge conjugation $(\mathcal{C})$ and parity $(\mathcal{P})$. We list the transformation properties of the fermion bilinears in Table II. While the hermiticity condition does not impose any

\begin{tabular}{l|c|c|c|c|c|c|c|c}
\hline \hline & 1 & $\gamma_{5}$ & $\gamma_{\mu}$ & $\gamma_{\mu} \gamma_{5}$ & $\sigma_{\mu \nu}$ & $\epsilon_{\mu \nu \rho \sigma}$ & $\grave{\partial_{\mu}}$ & $\partial_{\mu}$ \\
\hline $\mathcal{P}$ & + & - & + & - & + & - & + & + \\
\hline $\mathcal{C}$ & + & + & - & + & - & + & - & + \\
\hline h.c. & + & - & + & + & + & + & - & + \\
\hline \hline
\end{tabular}

TABLE II: Transformation properties of the fermion bilinears (with the different elements of the Clifford algebra), Levi-Civita tensor, and derivative operators under parity $(\mathcal{P})$, charge conjugation $(\mathcal{C})$, and hermitian conjugation (h.c.). The symbol $\partial_{\mu}$ in the last column stands for the four-gradient acting on the whole fermion bilinear.

constraint, since one can always multiply the individual bilinears by appropriate factors of $i$, the $\mathcal{C}$ and $\mathcal{P}$ symmetries must be enforced.

Second, we observe that derivatives $\partial$ acting on the whole bilinear are of order $Q$, while derivatives $\overleftrightarrow{\partial}$ acting inside a bilinear are of order $Q^{0}$ due to the presence of the nucleon mass. Therefore, at each order in the power counting, only a finite number of $\partial$ appears, while it is possible to have, in principle, any number of $\overleftrightarrow{\partial}$. The situation is not so hopeless, however. For instance, the contracted product $\overleftrightarrow{\partial}_{\mu} \overleftrightarrow{\partial}^{\mu}$ inside a bilinear yields a squared mass term (without derivatives) plus a $\partial_{\mu} \partial^{\mu}$ acting on the whole bilinear, which is suppressed by $Q^{2}$. Similarly, a term like $\overleftrightarrow{\not \partial} \equiv \overleftrightarrow{\partial}_{\mu} \gamma^{\mu}$, resulting from the contraction, in a bilinear, of $\overleftrightarrow{\partial}_{\mu}$ with one of the elements of the Clifford algebra, can be replaced by a term without derivatives by making use of the equations of motion, i.e. $i \not \partial \psi=m \psi$ and its adjoint; for 
example,

$$
\bar{\psi} i \overleftrightarrow{\partial}_{\mu} \sigma^{\mu \nu} \psi=\bar{\psi}\left(\gamma^{\nu} \vec{\not}+\overleftarrow{\not \partial} \gamma^{\nu}\right) \psi-\partial^{\nu}(\bar{\psi} \psi)=-\partial^{\nu}(\bar{\psi} \psi)
$$

Hence, in general, no two Lorentz indices in a fermion bilinear can be contracted with one another, except for the Levi-Civita tensor and for the (suppressed) $\partial^{2}$ acting on the whole bilinear.

Some of the most problematic terms are of the type

$$
\widetilde{O}_{\Gamma_{A} \Gamma_{B}}^{(n)}=\frac{1}{(2 m)^{2 n}}\left(\bar{\psi} i \overleftrightarrow{\partial^{\mu_{1}}} i \overleftrightarrow{\partial^{\mu_{2}}} \ldots i \overleftrightarrow{\partial}^{\mu_{n}} \Gamma_{A}^{\alpha} \psi\right)\left(\bar{\psi} i \overleftrightarrow{\partial}_{\mu_{1}} i \overleftrightarrow{\partial}_{\mu_{2}} \ldots i \overleftrightarrow{\partial}_{\mu_{n}} \Gamma_{B \alpha} \psi\right)
$$

since, as stated above, $n$ can be any integer. In fact, a little thought shows that terms with $n>1$ do not introduce any new operator structure up to $\mathcal{O}\left(Q^{2}\right)$ included. This is most easily seen by considering the matrix elements of such terms between initial and final two-nucleon states with momenta, respectively, $\mathbf{p}_{1}, \mathbf{p}_{2}$ and $\mathbf{p}_{3}, \mathbf{p}_{4}$. These matrix elements consist of the product of two factors: one given (in a schematic notation) by $\left(\bar{u}_{3} \Gamma_{A}^{\alpha} u_{1}\right)\left(\bar{u}_{4} \Gamma_{B \alpha} u_{2}\right)$ - the $u_{i}$ denote Dirac spinors - and another involving the particles' four-momenta,

$$
\frac{\left[\left(p_{1}+p_{3}\right) \cdot\left(p_{2}+p_{4}\right)\right]^{n}}{(2 m)^{2 n}}
$$

which to $\mathcal{O}\left(Q^{2}\right)$ can be approximated as

$$
1+\frac{n}{4 m^{2}}\left[\mathbf{p}_{1}^{2}+\mathbf{p}_{2}^{2}+\mathbf{p}_{3}^{2}+\mathbf{p}_{4}^{2}-\left(\mathbf{p}_{1}+\mathbf{p}_{3}\right) \cdot\left(\mathbf{p}_{2}+\mathbf{p}_{4}\right)\right] .
$$

Therefore, as discussed in more detail in the next section, one only needs, in practice, to account for terms of type (2.3) with $n=0,1$.

\section{B. Lagrangian to order $Q^{2}$}

Following the criteria laid out in the previous section, a complete but non-minimal set consisting of $36 \mathcal{P}$ - and $\mathcal{C}$-conserving operators, denoted as $\widetilde{O}_{i}$, is obtained. They are listed in Table III, Note that some operator structures are missing, since they do not contribute at order $Q^{2}$. For instance, operators having the $1 \otimes \gamma_{5}$ structure are at least of order $Q^{4}: \mathcal{P}$ symmetry requires the presence of an $\epsilon^{\mu \nu \alpha \beta}$ whose indices (three of which space-like) must be contracted with partial derivatives, and an additional factor $Q$ comes from the presence of $\gamma_{5}$, which mixes large and small components of the Dirac spinors.

The non-relativistic reduction of the $\widetilde{O}_{i}$ up to terms of order $Q^{2}$ included is tedious but straightforward. To this end, the relativistic field (specifically, its positive-energy component, where the $(+)$ superscript has been dropped for simplicity)

$$
\psi(x)=\int \frac{d \mathbf{p}}{(2 \pi)^{3}} \frac{m}{E_{p}} \widetilde{b}_{s}(\mathbf{p}) u^{(s)}(\mathbf{p}) \mathrm{e}^{-i p \cdot x}
$$

with normalizations

$$
\left[\widetilde{b}_{s}(\mathbf{p}), \widetilde{b}_{s^{\prime}}^{\dagger}\left(\mathbf{p}^{\prime}\right)\right]_{+}=\frac{E_{p}}{m}(2 \pi)^{3} \delta\left(\mathbf{p}-\mathbf{p}^{\prime}\right) \delta_{s s^{\prime}}, \quad \bar{u}^{(s)}(\mathbf{p}) u^{\left(s^{\prime}\right)}(\mathbf{p})=\delta_{s s^{\prime}}
$$




\begin{tabular}{|c|c|c|}
\hline $1 \otimes 1$ & $\begin{array}{l}\widetilde{O}_{1} \\
\widetilde{O}_{2} \\
\widetilde{O}_{3}\end{array}$ & $\begin{array}{c}\frac{(\bar{\psi} \psi)(\bar{\psi} \psi)}{4 m^{2}}(\bar{\psi} i \stackrel{\leftrightarrow}{\partial} \mu \psi)\left(\bar{\psi} i \overleftrightarrow{\partial}_{\mu} \psi\right) \\
\frac{1}{4 m^{2}}(\bar{\psi} \psi) \partial^{2}(\bar{\psi} \psi)\end{array}$ \\
\hline $1 \otimes \gamma$ & $\begin{array}{l}\widetilde{O}_{4} \\
\widetilde{O}_{5} \\
\widetilde{O}_{6}\end{array}$ & 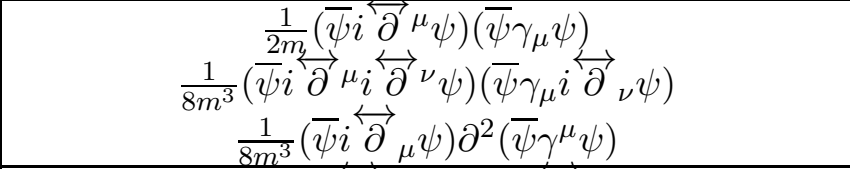 \\
\hline $1 \otimes \gamma \gamma_{5}$ & $\widetilde{O}_{7}$ & $\frac{1}{8 m^{3}} \epsilon_{\mu \nu \alpha \beta}\left(\bar{\psi} i \vec{\partial}^{\mu} \psi\right) \partial^{\nu}\left(\bar{\psi} i \vec{\partial}^{\alpha} \gamma^{\beta} \gamma_{5} \psi\right)$ \\
\hline$\gamma_{5} \otimes \gamma_{5}$ & $\widetilde{O}_{8}$ & $\left(\bar{\psi} i \gamma_{5} \psi\right)\left(\bar{\psi} i \gamma_{5} \psi\right)$ \\
\hline$\gamma_{5} \otimes \sigma$ & $\begin{array}{l}\widetilde{O}_{9} \\
\widetilde{O}_{10} \\
\end{array}$ & $\begin{array}{l}\frac{1}{4 m^{2}} \epsilon_{\mu \nu \alpha \beta}\left(\bar{\psi} i \gamma_{5} \psi\right) \partial^{\mu}\left(\bar{\psi} i \overleftrightarrow{\partial^{\nu}} \sigma^{\alpha \beta} \psi\right) \\
\frac{1}{4 m^{2}} \epsilon_{\mu \nu \alpha \beta}\left(\bar{\psi} i \gamma_{5} i \overleftrightarrow{\partial^{\mu}} \mu\right) \partial^{\nu}\left(\bar{\psi} \sigma^{\alpha \beta} \psi\right)\end{array}$ \\
\hline$\gamma \otimes \gamma$ & $\begin{array}{l}\widetilde{O}_{11} \\
\widetilde{O}_{12} \\
\widetilde{O}_{13} \\
\widetilde{O}_{14} \\
\widetilde{O}_{15} \\
\end{array}$ & 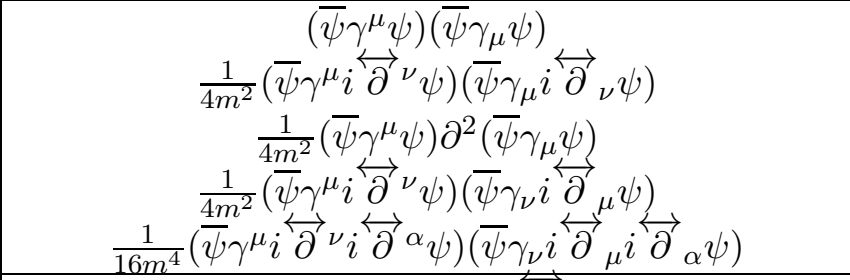 \\
\hline$\gamma \otimes \gamma \gamma_{5}$ & $\begin{array}{l}\widetilde{O}_{16} \\
\widetilde{O}_{17} \\
\widetilde{O}_{18} \\
\end{array}$ & 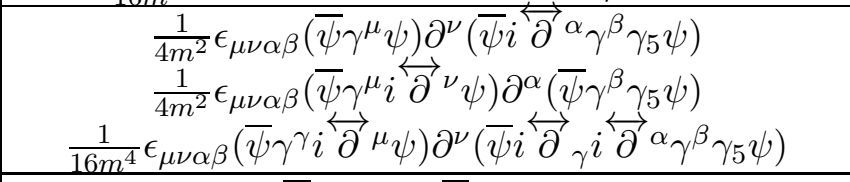 \\
\hline$\gamma \gamma_{5} \otimes \gamma \gamma_{5}$ & $\begin{array}{l}\widetilde{O}_{19} \\
\widetilde{O}_{20} \\
\widetilde{O}_{21} \\
\widetilde{O}_{22} \\
\end{array}$ & 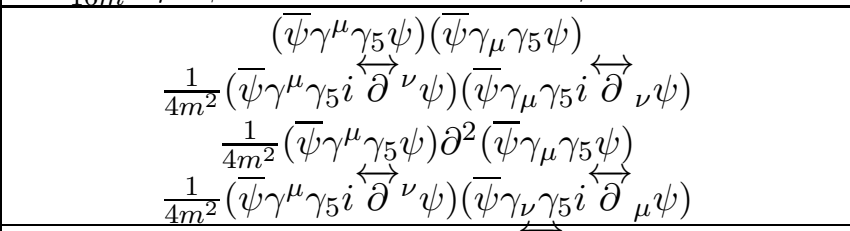 \\
\hline$\gamma \gamma_{5} \otimes \sigma$ & $\begin{array}{l}\widetilde{O}_{23} \\
\widetilde{O}_{24} \\
\widetilde{O}_{25} \\
\widetilde{O}_{26} \\
\widetilde{O}_{27} \\
\widetilde{O}_{28} \\
\widetilde{O}_{29} \\
\widetilde{O}_{30} \\
\widetilde{O}_{31} \\
\widetilde{O}_{32} \\
\widetilde{O}_{33} \\
\widetilde{O}_{34} \\
\widetilde{O}_{35} \\
\widetilde{O}_{36}\end{array}$ & 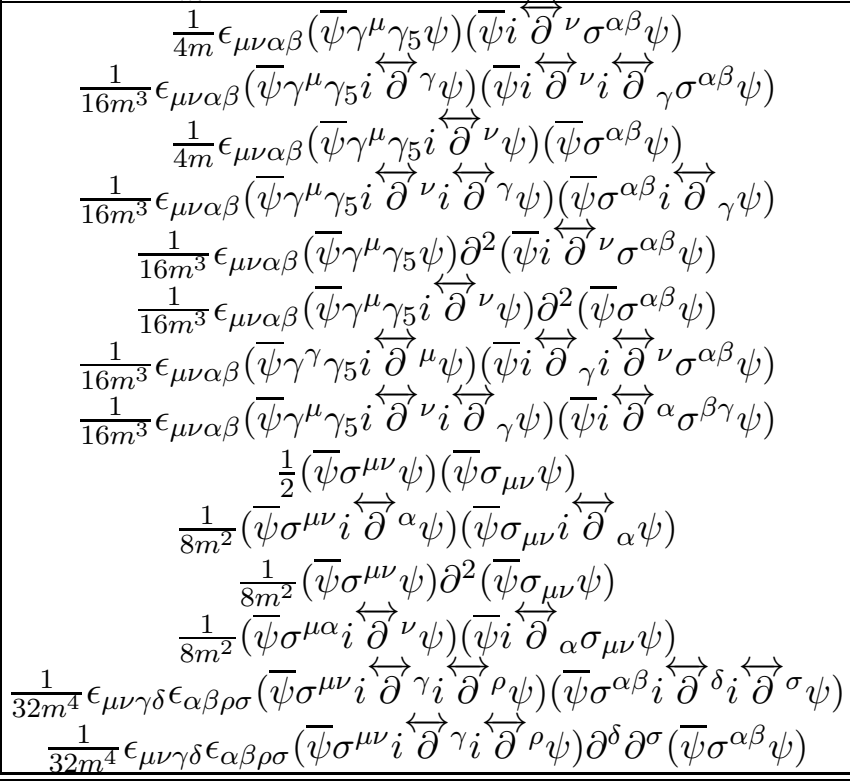 \\
\hline
\end{tabular}

TABLE III: A complete, but non-minimal, set of relativistic contact interactions. Note that the field operator products are understood to be normal-ordered. 
is expanded in terms of the non-relativistic field $N(x)$, defined in Eq. (1.2), as

$$
\psi(x)=\left[\left(\begin{array}{l}
1 \\
0
\end{array}\right)-\frac{i}{2 m}\left(\begin{array}{c}
0 \\
\boldsymbol{\sigma} \cdot \boldsymbol{\nabla}
\end{array}\right)+\frac{1}{8 m^{2}}\left(\begin{array}{c}
\boldsymbol{\nabla}^{2} \\
0
\end{array}\right)\right] N(x)+\mathcal{O}\left(Q^{3}\right) .
$$

Note that the relativistic $(\widetilde{b})$ and non-relativistic $(b)$ versions of the annihilation operator are related to each other by $b_{s}(\mathbf{p})=\sqrt{m / E_{p}} \widetilde{b}_{s}(\mathbf{p})$. Partial integrations and use of the fields' equations of motion to eliminate time derivatives,

$$
\begin{aligned}
\frac{i}{2 m} \vec{\psi}^{\overleftrightarrow{\partial}} \psi & =\psi^{\dagger}\left[1-\frac{i}{2 m} \gamma \cdot(\overleftarrow{\nabla}+\vec{\nabla})\right] \psi \\
& =N^{\dagger} N-\frac{1}{8 m^{2}} N^{\dagger}\left[(\overleftarrow{\nabla}+\vec{\nabla})^{2}+2 i \boldsymbol{\sigma} \cdot \overleftarrow{\nabla} \times \vec{\nabla}\right] N+\mathcal{O}\left(Q^{3}\right)
\end{aligned}
$$

lead to the operators $\widetilde{O}_{i}^{\text {NR }}$ of Table IV. They are given there as linear combinations of the operator basis $O_{i}$, defined previously (see Table $\amalg$ ).

Returning briefly to the discussion of the terms of type $\widetilde{O}_{\Gamma_{A} \Gamma_{B}}^{(n)}$ in Eq. (2.3), it is useful to separate such terms into three classes, depending on whether the non-relativistic expansion of the spinor matrix element $\left(\bar{u}_{3} \Gamma_{A}^{\alpha} u_{1}\right)\left(\bar{u}_{4} \Gamma_{B \alpha} u_{2}\right)$ is i) $1+\mathcal{O}\left(Q^{2}\right)$ (class I), or ii) $\pm \boldsymbol{\sigma}_{1} \cdot \boldsymbol{\sigma}_{2}+\mathcal{O}\left(Q^{2}\right)$ (class II), or iii) $\mathcal{O}\left(Q^{2}\right)$ (class III). Making use of the relations (2.8) and (2.9), we find that terms in class I reduce to

$$
\widetilde{O}_{\Gamma_{A} \Gamma_{B}}^{(n)}=\widetilde{O}_{\Gamma_{A} \Gamma_{B}}^{(n=0)}+\frac{n}{4 m^{2}}\left(O_{1}-2 O_{2}-2 O_{3}\right)+\mathcal{O}\left(Q^{4}\right)
$$

while those in class II reduce to

$$
\widetilde{O}_{\Gamma_{A} \Gamma_{B}}^{(n)}=\widetilde{O}_{\Gamma_{A} \Gamma_{B}}^{(n=0)} \pm \frac{n}{4 m^{2}}\left(3 O_{9}+2 O_{12}+2 O_{14}\right)+\mathcal{O}\left(Q^{4}\right),
$$

and lastly the terms in class III are simply given, for any $n$, by $\widetilde{O}_{\Gamma_{A} \Gamma_{B}}^{(n=0)}$ up to corrections $\mathcal{O}\left(Q^{4}\right)$. A quick glance at Tables III and IV shows that the relations above are verified: consider, for example, $\widetilde{O}_{1}$ and $\widetilde{O}_{2}$ in class I, $\widetilde{O}_{20}$ and $\widetilde{O}_{21}$ in class II, and $\widetilde{O}_{8}$ in class III, and their corresponding non-relativistic reductions.

Inspection of Table IV shows that a set of linearly independent operator combinations can be defined as

$$
\begin{aligned}
& O_{S}+\left(O_{1}+O_{3}+O_{5}+O_{6}\right) /\left(4 m^{2}\right) \\
& O_{T}-\left(O_{5}+O_{6}-O_{7}+O_{8}+2 O_{12}+O_{14}\right) /\left(4 m^{2}\right) \\
& O_{1}+2 O_{2} \\
& 2 O_{2}+O_{3} \\
& O_{9}+2 O_{12} \\
& O_{9}+O_{14} \\
& O_{5}-O_{6} \\
& O_{7}+2 O_{10} \\
& O_{7}+O_{8}+2 O_{13}
\end{aligned}
$$

consisting of 2 leading (of order $Q^{0}$ ) and 7 sub-leading $\left(Q^{2}\right)$ ones, in agreement with the results of an analysis based on the heavy-baryon formulation of EFT [9, 14], so that the 


\begin{tabular}{|c|c|}
\hline $\begin{array}{l}\widetilde{O}_{1}^{\mathrm{NR}} \\
\widetilde{O}_{2}^{\mathrm{NR}} \\
\widetilde{O}_{3}^{\mathrm{NR}} \\
\end{array}$ & $\begin{array}{c}O_{S}+\frac{1}{4 m^{2}}\left(O_{1}+2 O_{2}+2 O_{3}+2 O_{5}\right) \\
O_{S}+\frac{1}{4 m^{2}}\left(2 O_{1}+2 O_{5}\right) \\
\frac{1}{4 m^{2}}\left(O_{1}+2 O_{2}\right)\end{array}$ \\
\hline$\widetilde{O}_{4}^{\mathrm{NR}}$ & $O_{S}+\frac{1}{4 m^{2}}\left(O_{1}-2 O_{2}+2 O_{6}\right)$ \\
\hline$\widetilde{O}_{5}^{\mathrm{NR}}$ & $O_{S}+\frac{1}{4 m^{2}}\left(2 O_{1}-4 O_{2}-2 O_{3}+2 O_{6}\right)$ \\
\hline$\widetilde{O}_{6}^{\mathrm{NR}}$ & $\frac{1}{4 m^{2}}\left(O_{1}+2 O_{2}\right)$ \\
\hline$\widetilde{O}_{7}^{\mathrm{NR}}$ & $\frac{1}{4 m^{2}}\left(-2 O_{5}+2 O_{6}\right)$ \\
\hline$\widetilde{O}_{8}^{\mathrm{NR}}$ & $\frac{1}{4 m^{2}}\left(O_{7}+2 O_{10}\right)$ \\
\hline$\widetilde{O}_{9}^{\mathrm{NR}}$ & $\frac{1}{4 m^{2}}\left(2 O_{7}+4 O_{10}\right)$ \\
\hline$\widetilde{O}_{10}^{\mathrm{NR}}$ & $\frac{1}{4 m^{2}}\left(-2 O_{7}-4 O_{10}\right)$ \\
\hline$\widetilde{O}_{11}^{\mathrm{NR}}$ & $O_{S}+\frac{1}{4 m^{2}}\left(-4 O_{2}-2 O_{5}+4 O_{6}+O_{7}-O_{9}+2 O_{10}-2 O_{12}\right)$ \\
\hline$\widetilde{O}_{12}^{\mathrm{NR}}$ & $O_{S}+\frac{1}{4 m^{2}}\left(O_{1}-6 O_{2}-2 O_{3}-2 O_{5}+4 O_{6}+O_{7}-O_{9}+2 O_{10}-2 O_{12}\right)$ \\
\hline$\widetilde{O}_{13}^{\mathrm{NR}}$ & $\frac{1}{4 m^{2}}\left(O_{1}+2 O_{2}\right)$ \\
\hline$\widetilde{O}_{14}^{\mathrm{NR}}$ & $O_{S}+\frac{1}{4 m^{2}}\left(O_{1}-6 O_{2}-2 O_{3}-2 O_{5}+4 O_{6}\right)$ \\
\hline$\widetilde{O}_{15}^{\mathrm{NR}}$ & $O_{S}+\frac{1}{4 m^{2}}\left(2 O_{1}-8 O_{2}-4 O_{3}-2 O_{5}+4 O_{6}\right)$ \\
\hline$\widetilde{O}_{16}^{\mathrm{NR}}$ & $\frac{1}{4 m^{2}}\left(-2 O_{5}+2 O_{6}+O_{7}-O_{9}+2 O_{10}-2 O_{12}\right)$ \\
\hline$\widetilde{O}_{17}^{\mathrm{NR}}$ & $\frac{1}{4 m^{2}}\left(-O_{7}+O_{9}-2 O_{10}+2 O_{12}\right)$ \\
\hline$\widetilde{O}_{18}^{\mathrm{NR}}$ & $\frac{1}{4 m^{2}}\left(-2 O_{5}+2 O_{6}\right)$ \\
\hline$\widetilde{O}_{19}^{\mathrm{NR}}$ & $-O_{T}-\frac{1}{4 m^{2}}\left(-2 O_{6}+O_{7}-O_{9}-2 O_{10}-2 O_{12}+2 O_{13}-2 O_{14}\right)$ \\
\hline$\widetilde{O}_{20}^{\mathrm{NR}}$ & $-O_{T}-\frac{1}{4 m^{2}}\left(-2 O_{6}+O_{7}+2 O_{9}-2 O_{10}+2 O_{13}\right)$ \\
\hline$\widetilde{O}_{21}^{\mathrm{NR}}$ & $\frac{1}{4 m^{2}}\left(-O_{9}-2 O_{12}\right)$ \\
\hline$\widetilde{O}_{22}^{\mathrm{NR}}$ & $\frac{1}{4 m^{2}}\left(-2 O_{7}-2 O_{8}-4 O_{13}\right)$ \\
\hline$\widetilde{O}_{23}^{\mathrm{NR}}$ & $-O_{T}-\frac{1}{4 m^{2}}\left(-2 O_{6}+2 O_{7}-O_{9}-2 O_{12}+2 O_{13}-2 O_{14}\right)$ \\
\hline$\widetilde{O}_{24}^{\mathrm{NR}}$ & $-O_{T}-\frac{1}{4 m^{2}}\left(-2 O_{6}+2 O_{7}+2 O_{9}+2 O_{13}\right)$ \\
\hline$\widetilde{O}_{25}^{\mathrm{NR}}$ & $-O_{T}-\frac{1}{4 m^{2}}\left(-2 O_{5}-2 O_{8}+O_{9}-2 O_{12}-2 O_{13}\right)$ \\
\hline$\widetilde{O}_{26}^{\mathrm{NR}}$ & $-O_{T}-\frac{1}{4 m^{2}}\left(-2 O_{5}-2 O_{8}+4 O_{9}-2 O_{13}+2 O_{14}\right)$ \\
\hline$\widetilde{O}_{27}^{\mathrm{NR}}$ & $\frac{1}{4 m^{2}}\left(-O_{9}-2 O_{12}\right)$ \\
\hline$\widetilde{O}_{28}^{\mathrm{NR}}$ & $\frac{1}{4 m^{2}}\left(-O_{9}-2 O_{12}\right)$ \\
\hline$\widetilde{O}_{29}^{\mathrm{NR}}$ & $\frac{1}{4 m^{2}}\left(-2 O_{7}-2 O_{8}-4 O_{13}\right)$ \\
\hline$\widetilde{O}_{30}^{\mathrm{NR}}$ & $\frac{1}{4 m^{2}}\left(-O_{5}+O_{6}-O_{7}-O_{8}+2 O_{9}-2 O_{13}+2 O_{14}\right)$ \\
\hline$\widetilde{O}_{31}^{\mathrm{NR}}$ & $O_{T}+\frac{1}{4 m^{2}}\left(-O_{1}-2 O_{2}-4 O_{5}+2 O_{6}+O_{7}-2 O_{8}+2 O_{10}-4 O_{12}-2 O_{13}\right)$ \\
\hline$\widetilde{O}_{32}^{\mathrm{NR}}$ & $O_{T}+\frac{1}{4 m^{2}}\left(-O_{1}-2 O_{2}-4 O_{5}+2 O_{6}+O_{7}-2 O_{8}+3 O_{9}+2 O_{10}-2 O_{12}-2 O_{13}+2 O_{14}\right)$ \\
\hline$\widetilde{O}_{33}^{\mathrm{NR}}$ & $\frac{1}{4 m^{2}}\left(O_{9}+2 O_{12}\right)$ \\
\hline$\widetilde{O}_{34}^{\mathrm{NR}}$ & $\frac{1}{4 m^{2}}\left(-\frac{1}{2} O_{1}-O_{2}-2 O_{5}+2 O_{6}-O_{7}-O_{8}+2 O_{9}-2 O_{13}+2 O_{14}\right)$ \\
\hline$\widetilde{O}_{35}^{N R}$ & $\frac{1}{4 m^{2}}\left(4 O_{7}+4 O_{8}+8 O_{13}\right)$ \\
\hline$\widetilde{O}_{36}^{N R}$ & $\frac{1}{4 m^{2}}\left(2 O_{7}+4 O_{10}\right)$ \\
\hline
\end{tabular}

TABLE IV: The non-relativistic expressions, up to order $Q^{2}$ included, corresponding to the contact interactions of Table III. 
effective Lagrangian can be written as

$$
\begin{aligned}
\mathcal{L}= & -\frac{1}{2} C_{S}\left[O_{S}+\frac{1}{4 m^{2}}\left(O_{1}+O_{3}+O_{5}+O_{6}\right)\right]-\frac{1}{2} C_{T}\left[O_{T}-\frac{1}{4 m^{2}}\left(O_{5}+O_{6}-O_{7}+O_{8}\right.\right. \\
& \left.\left.+2 O_{12}+O_{14}\right)\right]-\frac{1}{2} C_{1}\left(O_{1}+2 O_{2}\right)+\frac{1}{8} C_{2}\left(2 O_{2}+O_{3}\right)-\frac{1}{2} C_{3}\left(O_{9}+2 O_{12}\right) \\
& -\frac{1}{8} C_{4}\left(O_{9}+O_{14}\right)+\frac{1}{4} C_{5}\left(O_{6}-O_{5}\right)-\frac{1}{2} C_{6}\left(O_{7}+2 O_{10}\right)-\frac{1}{16} C_{7}\left(O_{7}+O_{8}+2 O_{13}\right) .
\end{aligned}
$$

Evaluation of the matrix elements of the operators $O_{i}$ between initial and final two-nucleon states with momenta $\mathbf{P} / 2+\mathbf{p}, \mathbf{P} / 2-\mathbf{p}$ and $\mathbf{P} / 2+\mathbf{p}^{\prime}, \mathbf{P} / 2-\mathbf{p}^{\prime}$, i.e.

$$
O_{i}\left(\mathbf{p}^{\prime}, \mathbf{p} ; \mathbf{P}\right)=\int d \mathbf{x}\left\langle\mathbf{P} / 2+\mathbf{p}^{\prime}, \mathbf{P} / 2-\mathbf{p}^{\prime}\left|O_{i}\right| \mathbf{P} / 2+\mathbf{p}, \mathbf{P} / 2-\mathbf{p}\right\rangle
$$

shows that the 7 sub-leading combinations above give vanishing $\mathbf{P}$-dependent contributions, and in fact lead, in the center-of-mass frame, to the $7 \mathbf{k}$ - and $\mathbf{K}$-dependent terms occurring in $v^{\mathrm{CT} 2}(\mathbf{k}, \mathbf{K})$, Eq. (1.6). Similarly, the 2 leading combinations and associated $1 / m^{2}$ corrections - first 2 lines of Eq. (2.12) - give rise, respectively, to the $\mathbf{P}$-dependent terms

$$
-\frac{P^{2}}{2 m^{2}}+\frac{i}{4 m^{2}}\left(\boldsymbol{\sigma}_{1}-\boldsymbol{\sigma}_{2}\right) \cdot \mathbf{P} \times \mathbf{k}
$$

and

$$
-\frac{P^{2}}{2 m^{2}} \boldsymbol{\sigma}_{1} \cdot \boldsymbol{\sigma}_{2}-\frac{i}{4 m^{2}}\left(\boldsymbol{\sigma}_{1}-\boldsymbol{\sigma}_{2}\right) \cdot \mathbf{P} \times \mathbf{k}+\frac{1}{m^{2}}\left(\boldsymbol{\sigma}_{1} \cdot \mathbf{P} \boldsymbol{\sigma}_{2} \cdot \mathbf{K}-\boldsymbol{\sigma}_{1} \cdot \mathbf{K} \boldsymbol{\sigma}_{2} \cdot \mathbf{P}\right),
$$

which, after multiplication by $C_{S} / 2$ and $C_{T} / 2$, are precisely the terms entering the potential $v_{\mathbf{P}}^{\mathrm{CT} 2}(\mathbf{k}, \mathbf{K})$ in Eq. (1.7).

\section{POINCARÉ ALGEBRA CONSTRAINTS}

As an alternative to the procedure discussed in the previous section, one can impose the Poincaré algebra constraints on the Hamiltonians derived from the Lagrangians in Eqs. (1.1) and (1.4), $\mathcal{H}_{I}^{(n)}=-\mathcal{L}_{I}^{(n)}$. In the instant form of relativistic dynamics, the interactions affect not only the Hamiltonian $H$ but also the boost generators $\mathbf{K}$. We write

$$
H=H_{0}+H_{I}, \quad \mathbf{K}=\mathbf{K}_{0}+\mathbf{K}_{I}, \quad \mathbf{P}=\mathbf{P}_{0}, \quad \mathbf{J}=\mathbf{J}_{0}
$$

to distinguish between the operators in the absence (with subscript 0) and in the presence (without subscript) of interactions, and impose the following commutation relations:

$$
\begin{aligned}
& {\left[J^{i}, J^{j}\right]=i \epsilon^{i j k} J^{k},\left[K^{i}, K^{j}\right]=-i \epsilon^{i j k} J^{k},\left[J^{i}, K^{j}\right]=i \epsilon^{i j k} K^{k},\left[P^{\mu}, P^{\nu}\right]=0} \\
& {\left[K^{i}, P^{j}\right]=i \delta^{i j} H,\left[J^{i}, P^{j}\right]=i \epsilon^{i j k} P^{k}, \quad\left[K^{i}, H\right]=i P^{i}, \quad\left[J^{i}, H\right]=0 .}
\end{aligned}
$$


The free Lorentz boost generators are derived from the energy-momentum tensor of the free fermionic theory $T^{\mu \nu}=(i / 2) \bar{\psi} \gamma^{\mu} \overleftrightarrow{\partial} \nu \psi$ as

$$
K_{0}^{i}=\int d \mathbf{x}\left(x^{i} T^{00}-t T^{0 i}\right)
$$

where, for the time being, $\psi$ denotes the field with both positive- and negative-energy components. The use of the symmetric energy-momentum tensor, the Belinfante tensor [15],

$$
\Theta^{\mu \nu}=T^{\mu \nu}+\frac{1}{8} \partial_{\alpha} \bar{\psi}\left[\gamma^{\alpha}, \sigma^{\mu \nu}\right]_{+} \psi
$$

would make no difference. Insertion of the field expansions in terms of normal modes in the equation above and manipulations of the resulting expressions lead to

$$
\begin{aligned}
K_{0}^{i}=\frac{i}{2} \int \frac{d \mathbf{p}}{(2 \pi)^{3}} \frac{m}{E_{p}}\left[E_{p}\left[\widetilde{b}_{s}^{\dagger}(\mathbf{p}) \overleftrightarrow{\nabla}_{\mathbf{p}}^{i} \widetilde{b}_{s}(\mathbf{p})\right]+m \widetilde{b}_{s}^{\dagger}(\mathbf{p}) \widetilde{b}_{s^{\prime}}(\mathbf{p})\left[u^{(s) \dagger}(\mathbf{p}) \overleftrightarrow{\nabla}_{\mathbf{p}}^{i} u^{\left(s^{\prime}\right)}(\mathbf{p})\right]\right. \\
\left.+E_{p}\left[\widetilde{d}_{s}^{\dagger}(\mathbf{p}) \overleftrightarrow{\nabla}_{\mathbf{p}}^{i} \widetilde{d}_{s}(\mathbf{p})\right]-m \widetilde{d}_{s^{\prime}}^{\dagger}(\mathbf{p}) \widetilde{d}_{s}(\mathbf{p})\left[v^{(s) \dagger}(\mathbf{p}) \overleftrightarrow{\nabla}_{\mathbf{p}}^{i} v^{\left(s^{\prime}\right)}(\mathbf{p})\right]\right]
\end{aligned}
$$

where $\widetilde{d}$ and $\widetilde{d}^{\dagger}$ are annihilation and creation operators for antinucleons, and $\nabla_{\mathbf{p}}^{i}$ denotes a derivative with respect to $p^{i}$. Note that $K_{0}^{i}$ is time independent, since it is the spatial integral of the time component $(\rho=0)$ of a conserved current, $\partial_{\rho} M^{\rho 0 i}=0$ with $M^{\rho \mu \nu}=$ $x^{\nu} \Theta^{\rho \mu}-x^{\mu} \Theta^{\rho \nu}$. By making use of

$$
\left[\mathbf{K}_{0}, \widetilde{b}_{s}(\mathbf{p})\right]=-i E_{p} \boldsymbol{\nabla}_{\mathbf{p}} \widetilde{b}_{s}(\mathbf{p})-\frac{1}{2\left(m+E_{p}\right)} \mathbf{p} \times \boldsymbol{\sigma}_{s s^{\prime}} \widetilde{b}_{s^{\prime}}(\mathbf{p})
$$

and a similar relation for $\widetilde{d}_{s}(\mathbf{p})$, in which the only difference is the sign of the second term on the right-hand-side of Eq. (3.6), one can show that the following commutation relations between the "free" generators are fulfilled

$$
\left[K_{0}^{i}, P^{j}\right]=i \delta^{i j} H_{0}, \quad\left[\mathbf{K}_{0}, H_{0}\right]=i \mathbf{P}
$$

where

$$
\left(\begin{array}{c}
\mathbf{P} \\
H_{0}
\end{array}\right)=\int \frac{d \mathbf{p}}{(2 \pi)^{3}} \frac{m}{E_{p}}\left(\begin{array}{c}
\mathbf{p} \\
E_{p}
\end{array}\right)\left[\widetilde{b}_{s}^{\dagger}(\mathbf{p}) \widetilde{b}_{s}(\mathbf{p})+\widetilde{d}_{s}^{\dagger}(\mathbf{p}) \widetilde{d}_{s}(\mathbf{p})\right]
$$

We now turn our attention to the interacting theory. The addition of an interaction term

$$
H_{I}=\int d \mathbf{x} \mathcal{H}_{I}(t=0, \mathbf{x})
$$

requires the addition of a corresponding term $\mathbf{K}_{I}$ in the boost generators, as inspection of the first commutator on the second line of Eq. (3.2) makes clear. Quite generally, this term can be expressed as $\mathbf{K}_{I}=\mathbf{W}+\delta \mathbf{W}$, where

$$
\mathbf{W}=\int d \mathbf{x} \mathbf{x} \mathcal{H}_{I}(0, \mathbf{x})
$$


and $\delta \mathbf{W}$ is translationally invariant, i.e. $\left[\delta W^{i}, P^{j}\right]=0$. This latter condition ensures that the commutator $\left[K^{i}, P^{j}\right]=i \delta^{i j} H$ is satisfied, since $\left[W^{i}, P^{j}\right]=i \delta^{i j} H_{I}$. A "minimal" choice would correspond to the case $\delta \mathbf{W}=0$ [7, 8].

In order to proceed systematically, it is useful to introduce the following low-energy power counting

$$
H_{0} \sim Q^{0}+\mathcal{O}\left(Q^{2}\right), \quad \mathbf{P} \sim Q, \quad \mathbf{J} \sim Q^{0}, \quad \mathbf{K}_{0} \sim Q^{-1}+\mathcal{O}\left(Q^{1}\right)
$$

which follows by observing that $\widetilde{b}$ and $\widetilde{b}^{\dagger}$, as well as their non-relativistic counterparts $b$ and $b^{\dagger}$, each scale as $Q^{-3 / 2}$, and by expanding $E_{p}$ and the Dirac spinors in powers of $p / m$. We now require that the commutation relations among the Poincaré group generators be satisfied order by order in this power counting. To this end, it is useful to express

$$
\mathbf{K}_{0}=\mathbf{K}_{0}^{(-1)}+\mathbf{K}_{0}^{(1)}+\ldots, \quad H_{0}=H_{0}^{(0)}+H_{0}^{(2)}+\ldots,
$$

where the superscript $(n)$ denotes the order in our power counting, that is $\mathbf{K}_{0}^{(n)}, H_{0}^{(n)} \sim Q^{n}$. Then the commutators of $\mathbf{K}_{0}^{(n)}$ and $H_{0}^{(n)}$ with the non-relativistic annihilation operator $b_{s}(\mathbf{p})$ read at leading order as

$$
\left[\mathbf{K}_{0}^{(-1)}, b_{s}(\mathbf{p})\right]=-i m \boldsymbol{\nabla}_{\mathbf{p}} b_{s}(\mathbf{p}), \quad\left[H_{0}^{(0)}, b_{s}(\mathbf{p})\right]=-m b_{s}(\mathbf{p}),
$$

and at next to leading order as

$$
\begin{aligned}
& {\left[\mathbf{K}_{0}^{(1)}, b_{s}(\mathbf{p})\right]=-i \frac{p^{2}}{2 m} \nabla_{\mathbf{p}} b_{s}(\mathbf{p})-\frac{1}{4 m} \mathbf{p} \times \boldsymbol{\sigma}_{s s^{\prime}} b_{s^{\prime}}(\mathbf{p})-i \frac{\mathbf{p}}{2 m} b_{s}(\mathbf{p}),} \\
& {\left[H_{0}^{(2)}, b_{s}(\mathbf{p})\right]=-\frac{p^{2}}{2 m} b_{s}(\mathbf{p})}
\end{aligned}
$$

where the last term in Eq. (3.14) comes from the gradient $\boldsymbol{\nabla}_{\mathbf{p}}$ acting on the factor $\sqrt{E_{p} / m}$ relating $\widetilde{b}_{s}(\mathbf{p})$ to $b_{s}(\mathbf{p})$. It can now be shown that only the sub-leading terms of $\left[K_{0}^{i}, K_{0}^{j}\right]$ and $\left[\mathbf{K}_{0}, H_{0}\right]$, respectively of order $Q^{0}$ and $Q^{1}$, are non-vanishing, consistently with the power counting for the angular momentum $(\mathbf{J})$ and linear momentum $(\mathbf{P})$ operators, established in Eq. (3.11).

We write the interaction Hamiltonian as

$$
H_{I}=H^{(3)}+H^{(5)}
$$

where $H^{(3)}$ and $H^{(5)}$ are obtained from the Lagrangians in Eqs. (1.1) and (1.4), and the superscripts denote the order in our power counting. Correspondingly, we have

$$
\mathbf{W}=\mathbf{W}^{(2)}+\mathbf{W}^{(4)}
$$

Assuming, for the time being, $\delta \mathbf{W}=0$, the relations to satisfy are

$$
\begin{gathered}
{\left[K_{0}^{i}+W^{(2) i}+W^{(4) i}+\ldots, K_{0}^{j}+W^{(2) j}+W^{(4) j}+\ldots\right]=-i \epsilon_{i j k} J^{k}=\left[K_{0}^{i}, K_{0}^{j}\right]} \\
{\left[\mathbf{K}_{0}+\mathbf{W}^{(2)}+\mathbf{W}^{(4)}+\ldots, H_{0}+H^{(3)}+H^{(5)}+\ldots\right]=i \mathbf{P}=\left[\mathbf{K}_{0}, H_{0}\right]}
\end{gathered}
$$

where the ... represent additional terms to be determined below. These relations impose non trivial constraints on $H^{(3)}$ and $H^{(5)}$. We first examine those on $H^{(3)}$. 
By expanding $\mathbf{K}_{0}$ and $H_{0}$ as in Eq. (3.12), we find that the leading order relations

$$
\begin{aligned}
{\left[K_{0}^{(-1) i}, W^{(2) j}\right]+\left[W^{(2) i}, K_{0}^{(-1) j}\right] } & =0, \\
{\left[\mathbf{K}_{0}^{(-1)}, H^{(3)}\right]+\left[\mathbf{W}^{(2)}, H_{0}^{(0)}\right] } & =0,
\end{aligned}
$$

are fulfilled (see Appendix $(\mathbb{A}$ ), so that inclusion of the (leading) contact Hamiltonian

$$
H^{(3)}=\frac{1}{2} \int d \mathbf{x}\left(C_{S} O_{S}+C_{T} O_{T}\right) \equiv C_{S} H_{S}^{(3)}+C_{T} H_{T}^{(3)}
$$

does not spoil the Poincaré covariance of the theory (in leading order) - the operators $O_{S}$, $O_{T}$, and $O_{i}$ are those defined in Table $\square$. At next-to-leading order, we may split $H^{(5)}$ as

$$
H^{(5)}=H_{1}^{(5)}+H_{2}^{(5)},
$$

where $H_{1}^{(5)}$ and the corresponding $\mathbf{W}_{1}^{(4)}$ are found by imposing the relations

$$
\begin{aligned}
{\left[K_{0}^{(1) i}, W^{(2) j}\right]+\left[W^{(2) i}, K_{0}^{(1) j}\right]+\left[K_{0}^{(-1) i}, W_{1}^{(4) j}\right]+\left[W_{1}^{(4) i}, K_{0}^{(-1) j}\right] } & =0, \\
{\left[\mathbf{K}_{0}^{(1)}, H^{(3)}\right]+\left[\mathbf{K}_{0}^{(-1)}, H_{1}^{(5)}\right]+\left[\mathbf{W}^{(2)}, H_{0}^{(2)}\right]+\left[\mathbf{W}_{1}^{(4)}, H_{0}^{(0)}\right] } & =0 .
\end{aligned}
$$

After some algebra (see Appendix (A), we find that

$$
H_{1}^{(5)}=C_{S} H_{S}^{(5)}+C_{T} H_{T}^{(5)}
$$

with

$$
\begin{aligned}
& H_{S}^{(5)}=\frac{1}{8 m^{2}} \int d \mathbf{x}\left(O_{1}+O_{3}+O_{5}+O_{6}\right) \\
& H_{T}^{(5)}=-\frac{1}{8 m^{2}} \int d \mathbf{x}\left(O_{5}+O_{6}-O_{7}+O_{8}+2 O_{12}+O_{14}\right) .
\end{aligned}
$$

The constraints involving $H_{2}^{(5)}$ and the corresponding $\mathbf{W}_{2}^{(4)}$,

$$
\begin{aligned}
{\left[K_{0}^{(-1) i}, W_{2}^{(4) j}\right]+\left[W_{2}^{(4) i}, K_{0}^{(-1) j}\right] } & =0, \\
{\left[\mathbf{K}_{0}^{(-1)}, H_{2}^{(5)}\right]+\left[\mathbf{W}_{2}^{(4)}, H_{0}^{(0)}\right] } & =0,
\end{aligned}
$$

are fulfilled as long as the Hamiltonian $H_{2}^{(5)}$ is constructed out of the 7 sub-leading operators listed at the end of Sec. IIB, or combinations thereof. This is also shown in Appendix A, The Hamiltonian $H_{1}^{(5)}+H_{2}^{(5)}$ leads to the $\mathbf{P}$-dependent and $\mathbf{P}$-independent potentials in Eqs. (1.6) and (1.7), in accordance with the derivation presented in Sec. III,

In closing, we note that, although these constraints correspond to the "minimal choice" $\delta \mathbf{W}=0$, the result holds in the general case. Indeed, the requirement that $\delta \mathbf{W}$ commute with the three-momentum operator implies that it be constructed as a spatial integral of fields and their derivatives only. No factors of $\mathbf{x}$, which would lower the counting power, are allowed inside the integral. The minimal power, in our counting, of an interacting (twobody) boost operator $\delta \mathbf{W}$ is therefore 3 , but is actually 4 if the relations (3.18)-(3.25) have to be fulfilled order by order. As a result, the only contributions of $\delta \mathbf{W}$ to Eqs. (3.24) and (3.25) are given by $\left[\delta W^{(4) i}, K_{0}^{(-1) j}\right]$ and $\left[\delta \mathbf{W}^{(4)}, H_{0}^{(0)}\right]$, both of which vanish, since $\delta \mathbf{W}$, being hermitian, must contain an equal number of creation and annihilation operators. 


\section{Acknowledgments}

One of the authors (R.S.) would like to thank the Physics Department of the University of Pisa, the INFN Pisa branch, and especially the Pisa group for the support and warm hospitality extended to him on several occasions. The work of R.S. is supported by the U.S. Department of Energy, Office of Nuclear Physics, under contract DE-AC05-06OR23177.

\section{Appendix A: Constraints on $H^{(3)}$ and $H^{(5)}$}

In this appendix we outline the derivation of the leading- and next-to-leading order relations in Eqs. (3.20)-(3.21) and Eqs. (3.24)-(3.25) involving $H^{(3)}$, as well as of the leading order relations in Eqs. (3.29)-(3.30) involving $H^{(5)}$. For brevity, we suppress spin indices, and introduce the notation $\boldsymbol{\nabla}_{k} \equiv \boldsymbol{\nabla}_{\mathbf{p}_{k}}, b_{k} \equiv b_{s_{k}}\left(\mathbf{p}_{k}\right)$, and

$$
\int_{\mathbf{p}} \equiv \int \mathrm{e}^{-i\left(\mathbf{p}_{1}-\mathbf{p}_{2}+\mathbf{p}_{3}-\mathbf{p}_{4}\right) \cdot \mathbf{x}} \prod_{k=1}^{4} \frac{d \mathbf{p}_{k}}{(2 \pi)^{3}}
$$

Consider the commutator

$$
\left[K_{0}^{(-1) i}, W^{(2) j}\right]=\left[K_{0}^{(-1) i}, C_{S} W_{S}^{(2) j}+C_{T} W_{T}^{(2) j}\right]
$$

where the terms $\mathbf{W}_{S}^{(2)}$ and $\mathbf{W}_{T}^{(2)}$ correspond to the interactions $H_{S}^{(3)}$ and $H_{T}^{(3)}$ [see Eq. (3.22)], for example

$$
W_{S}^{(2)}=\frac{1}{2} \int d \mathbf{x} \mathbf{x}\left(N^{\dagger} N\right)\left(N^{\dagger} N\right)
$$

Making use of

$$
\left[\mathbf{K}_{0}^{(-1)}, b_{1}^{\dagger} b_{2} b_{3}^{\dagger} b_{4}\right]=-i m\left(\sum_{k=1}^{4} \nabla_{k}\right) b_{1}^{\dagger} b_{2} b_{3}^{\dagger} b_{4}
$$

we find that

$$
\left[K_{0}^{(-1) i}, W_{S}^{(2) j}\right]=-i \frac{m}{2} \int d \mathbf{x} x^{j} \int_{\mathbf{p}}\left(\sum_{k=1}^{4} \nabla_{k}^{i}\right) b_{1}^{\dagger} b_{2} b_{3}^{\dagger} b_{4}
$$

which vanishes after partial integrations with respect to the $\mathbf{p}_{k}$ 's-note the exponential factor in Eq. (A1). The terms involving $\mathbf{W}_{T}^{(2)}$ as well as those occurring in $\left[\mathbf{K}_{0}^{(-1)}, H^{(3)}\right]$ can be worked out similarly, while those in $\left[\mathbf{W}^{(2)}, H_{0}^{(0)}\right]$ vanish, since

$$
\left[b_{1}^{\dagger} b_{2} b_{3}^{\dagger} b_{4}, H_{0}^{(0)}\right]=0 \text {. }
$$

Thus each of the commutators entering the leading order relations vanishes.

Moving on to the next-to-leading order relations, consider first $H_{S}^{(3)}$. We obtain

$$
\left[\mathbf{K}_{0}^{(1)}, H_{S}^{(3)}\right]=\frac{1}{2 m} \int d \mathbf{x} \int_{\mathbf{p}}\left[\left[i\left(\mathbf{p}_{1}+\mathbf{p}_{2}\right)+\left(p_{1}^{2}-p_{2}^{2}\right) \mathbf{x}\right] b_{1}^{\dagger} b_{2} b_{3}^{\dagger} b_{4}+b_{1}^{\dagger} \frac{\mathbf{p}_{1}-\mathbf{p}_{2}}{2} \times \boldsymbol{\sigma} b_{2} b_{3}^{\dagger} b_{4}\right],
$$


where the terms involving the momenta $\mathbf{p}_{3}$ and $\mathbf{p}_{4}$ reduce to those with $\mathbf{p}_{1}$ and $\mathbf{p}_{2}$ after exchanging $3 \rightleftharpoons 1$ and $4 \rightleftharpoons 2$. The linear term in $\mathbf{x}$ is canceled by $\left[\mathbf{W}_{S}^{(2)}, H_{0}^{(2)}\right]$. In order to cancel the rest, one requires an interaction term $H_{S}^{(5)}$, given by

$$
\begin{aligned}
H_{S}^{(5)}= & -\frac{1}{8 m^{2}} \int d \mathbf{x} \int_{\mathbf{p}}\left[\left(p_{1}^{2}+p_{2}^{2}+\mathbf{p}_{2} \cdot \mathbf{p}_{4}+\mathbf{p}_{1} \cdot \mathbf{p}_{3}\right) b_{1}^{\dagger} b_{2} b_{3}^{\dagger} b_{4}\right. \\
& \left.+i b_{1}^{\dagger}\left(\mathbf{p}_{1} \times \mathbf{p}_{2}-\mathbf{p}_{3} \times \mathbf{p}_{4}\right) \cdot \boldsymbol{\sigma} b_{2} b_{3}^{\dagger} b_{4}\right] \\
= & \frac{1}{8 m^{2}} \int d \mathbf{x}\left[\left(N^{\dagger} \overleftarrow{\nabla}{ }^{2} N+N^{\dagger} \vec{\nabla}^{2} N\right)\left(N^{\dagger} N\right)+\left(N^{\dagger} \vec{\nabla} N\right)^{2}+\left(N^{\dagger} \overleftarrow{\nabla} N\right)^{2}\right. \\
& \left.+i\left(N^{\dagger} \overleftarrow{\nabla} \cdot \boldsymbol{\sigma} \times \vec{\nabla} N\right)\left(N^{\dagger} N\right)+i\left(N^{\dagger} \boldsymbol{\sigma} N\right) \cdot\left(N^{\dagger} \overleftarrow{\nabla} \times \vec{\nabla} N\right)\right]
\end{aligned}
$$

and a corresponding $\mathbf{W}_{S}^{(4)}$, which, however, commutes with $H_{0}^{(0)}$. Proceeding in a similar fashion for $H_{T}^{(3)}$, one finds that an interaction term $H_{T}^{(5)}$,

$$
\begin{aligned}
H_{T}^{(5)}= & -\frac{1}{8 m^{2}} \int d \mathbf{x} \int_{\mathbf{p}}\left[\left[2 \delta^{j k} \mathbf{p}_{2} \cdot\left(\mathbf{p}_{1}+\mathbf{p}_{3}\right)+p_{2}^{j} p_{4}^{k}-p_{2}^{k} p_{4}^{j}+p_{1}^{j} p_{3}^{k}-p_{1}^{k} p_{3}^{j}\right] b_{1}^{\dagger} \sigma^{j} b_{2} b_{3}^{\dagger} \sigma^{k} b_{4}\right. \\
& \left.+i b_{1}^{\dagger}\left(\mathbf{p}_{3} \times \mathbf{p}_{4}-\mathbf{p}_{1} \times \mathbf{p}_{2}\right) \cdot \boldsymbol{\sigma} b_{2} b_{3}^{\dagger} b_{4}\right] \\
= & -\frac{1}{8 m^{2}} \int d \mathbf{x}\left[-\left(N^{\dagger} \boldsymbol{\sigma} \cdot \vec{\nabla} N\right)\left(N^{\dagger} \boldsymbol{\sigma} \cdot \vec{\nabla} N\right)-\left(N^{\dagger} \boldsymbol{\sigma} \cdot \overleftarrow{\nabla} N\right)\left(N^{\dagger} \boldsymbol{\sigma} \cdot \overleftarrow{\nabla} N\right)\right. \\
& +\left(N^{\dagger} \sigma^{j} \overrightarrow{\nabla^{k}} N\right)\left(N^{\dagger} \sigma^{k} \vec{\nabla}^{j} N\right)+\left(N^{\dagger} \sigma^{j} \overleftarrow{\nabla^{k}} N\right)\left(N^{\dagger} \sigma^{k} \overleftarrow{\nabla^{j}} N\right) \\
& +2\left(N^{\dagger} \sigma^{j} \overrightarrow{\nabla^{k}} N\right)\left(N^{\dagger} \overleftarrow{\nabla^{k}} \sigma^{j} N\right)+2\left(N^{\dagger} \overleftarrow{\nabla} \sigma^{j} \cdot \vec{\nabla} N\right)\left(N^{\dagger} \sigma^{j} N\right) \\
& \left.+i\left(N^{\dagger} \overleftarrow{\nabla} \cdot \boldsymbol{\sigma} \times \vec{\nabla} N\right)\left(N^{\dagger} N\right)+i\left(N^{\dagger} \boldsymbol{\sigma} N\right) \cdot\left(N^{\dagger} \overleftarrow{\nabla} \times \vec{\nabla} N\right)\right]
\end{aligned}
$$

and a corresponding boost operator $\mathbf{W}_{T}^{(4)}$, are required in order to satisfy the $T$-piece of the commutators. Thus Eq. (3.25) holds. Similarly, Eq. (3.24) can also be shown to hold. The expressions for $H_{S}^{(5)}$ and $H_{T}^{(5)}$ correspond to those listed in Eqs. (3.27)-(3.28).

We now turn our attention to the constraint on $H_{2}^{(5)}$ implied by Eq. (3.30). We first observe that the commutator $\left[\mathbf{W}_{2}^{(4)}, H_{0}^{(0)}\right]$ vanishes. Defining

$$
\left[O_{i}\right] \equiv \int d \mathbf{x}\left[\mathbf{K}_{0}^{(-1)}, O_{i}\right]
$$

we find

$$
\begin{aligned}
& {\left[O_{1}\right]=-2 i m \int d \mathbf{x} \int_{\mathbf{p}}\left(\mathbf{p}_{1}+\mathbf{p}_{2}\right) b_{1}^{\dagger} b_{2} b_{3}^{\dagger} b_{4},} \\
& {\left[O_{2}\right]=i m \int d \mathbf{x} \int_{\mathbf{p}}\left(\mathbf{p}_{1}+\mathbf{p}_{2}\right) b_{1}^{\dagger} b_{2} b_{3}^{\dagger} b_{4},} \\
& {\left[O_{3}\right]=-2 i m \int d \mathbf{x} \int_{\mathbf{p}}\left(\mathbf{p}_{1}+\mathbf{p}_{2}\right) b_{1}^{\dagger} b_{2} b_{3}^{\dagger} b_{4}} \\
& {\left[O_{4}\right]=0}
\end{aligned}
$$




$$
\begin{aligned}
{\left[O_{5}\right] } & =-m \int d \mathbf{x} \int_{\mathbf{p}}\left(\mathbf{p}_{1}-\mathbf{p}_{2}\right) \times b_{1}^{\dagger} \boldsymbol{\sigma} b_{2} b_{3}^{\dagger} b_{4} \\
{\left[O_{6}\right] } & =m \int d \mathbf{x} \int_{\mathbf{p}}\left(\mathbf{p}_{3}-\mathbf{p}_{4}\right) \times b_{1}^{\dagger} \boldsymbol{\sigma} b_{2} b_{3}^{\dagger} b_{4} \\
{\left[O_{7}\right] } & =-2 i m \int d \mathbf{x} \int_{\mathbf{p}} b_{1}^{\dagger} \boldsymbol{\sigma} b_{2} b_{3}^{\dagger}\left(\mathbf{p}_{3}+\mathbf{p}_{4}\right) \cdot \boldsymbol{\sigma} b_{4} \\
{\left[O_{8}\right] } & =-2 i m \int d \mathbf{x} \int_{\mathbf{p}} b_{1}^{\dagger} \boldsymbol{\sigma} b_{2} b_{3}^{\dagger}\left(\mathbf{p}_{1}+\mathbf{p}_{2}\right) \cdot \boldsymbol{\sigma} b_{4} \\
{\left[O_{9}\right] } & =-2 i m \int d \mathbf{x} \int_{\mathbf{p}}\left(\mathbf{p}_{1}+\mathbf{p}_{2}\right) b_{1}^{\dagger} \boldsymbol{\sigma} b_{2} \cdot b_{3}^{\dagger} \boldsymbol{\sigma} b_{4} \\
{\left[O_{10}\right] } & =i m \int d \mathbf{x} \int_{\mathbf{p}} b_{1}^{\dagger} \boldsymbol{\sigma} b_{2} b_{3}^{\dagger}\left(\mathbf{p}_{3}+\mathbf{p}_{4}\right) \cdot \boldsymbol{\sigma} b_{4} \\
{\left[O_{11}\right] } & =i m \int d \mathbf{x} \int_{\mathbf{p}} b_{1}^{\dagger} \boldsymbol{\sigma} b_{2} b_{3}^{\dagger}\left(\mathbf{p}_{1}+\mathbf{p}_{2}\right) \cdot \boldsymbol{\sigma} b_{4} \\
{\left[O_{12}\right] } & =i m \int d \mathbf{x} \int_{\mathbf{p}}\left(\mathbf{p}_{1}+\mathbf{p}_{2}\right) b_{1}^{\dagger} \boldsymbol{\sigma} b_{2} \cdot b_{3}^{\dagger} \boldsymbol{\sigma} b_{4} \\
{\left[O_{13}\right] } & =i m \int d \mathbf{x} \int_{\mathbf{p}} b_{1}^{\dagger} \boldsymbol{\sigma} b_{2} b_{3}^{\dagger}\left(\mathbf{p}_{1}+\mathbf{p}_{2}+\mathbf{p} \mathbf{p}_{3}+\mathbf{p}_{4}\right) \cdot \boldsymbol{\sigma} b_{4} \\
{\left[O_{14}\right] } & =2 i m \int d \mathbf{x} \int_{\mathbf{p}}\left(\mathbf{p}_{1}+\mathbf{p}_{2}\right) b_{1}^{\dagger} \boldsymbol{\sigma} b_{2} \cdot b_{3}^{\dagger} \boldsymbol{\sigma} b_{4}
\end{aligned}
$$

and only 7 combinations of these operators satisfy the constraint in Eq. (3.30), such as those in Eq. (2.12). It is possible to show that these 7 combinations also satisfy the constraint of Eq. (3.29).

[1] S. Weinberg, Phys. Lett. B 251, 288 (1990); Nucl. Phys. B 363, 3 (1991); Phys. Lett. B 295, 114 (1992).

[2] V. Bernard, N. Kaiser and U. G. Meissner, Int. J. Mod. Phys. E 4, 193 (1995); U. van Kolck, Prog. Part. Nucl. Phys. 43, 337 (1999); P. F. Bedaque and U. van Kolck, Ann. Rev. Nucl. Part. Sci. 52, 339 (2002); E. Epelbaum, Prog. Part. Nucl. Phys. 57, 654 (2006); E. Epelbaum, H. W. Hammer and U. G. Meissner, Rev. Mod. Phys. 81, 1773 (2009).

[3] C. Ordonez, L. Ray and U. van Kolck, Phys. Rev. C 53, 2086 (1996).

[4] S. Pastore, L. Girlanda, R. Schiavilla, M. Viviani and R. B. Wiringa, Phys. Rev. C 80, 034004 (2009).

[5] E. Epelbaum, W. Gloeckle and U. G. Meissner, Nucl. Phys. A 637, 107 (1998).

[6] P. A. M. Dirac, Rev. Mod. Phys. 21, 392 (1949).

[7] R. A. Krajcik and L. L. Foldy, Phys. Rev. D 10, 1777 (1974); J. L. Friar, Phys. Rev. C 12, 695 (1975).

[8] J. Carlson, V. R. Pandharipande and R. Schiavilla, Phys. Rev. C 47, 484 (1993); J. L. Forest, V. R. Pandharipande, J. Carlson and R. Schiavilla, Phys. Rev. C 52, 576 (1995).

[9] E. Epelbaum, Ph.D. thesis (Bochum, 2000) unpublished; E. Epelbaum, U. G. Meissner, W. Gloeckle and C. Elster, Phys. Rev. C 65, 044001 (2002) arXiv:nucl-th/0106007. 
[10] H. Georgi, Phys. Lett. B 240, 447 (1990); E. E. Jenkins and A. V. Manohar, Phys. Lett. B 255, 558 (1991); V. Bernard, N. Kaiser, J. Kambor and U. G. Meissner, Nucl. Phys. B 388, 315 (1992).

[11] M. E. Luke and A. V. Manohar, Phys. Lett. B 286, 348 (1992).

[12] N. Brambilla, D. Gromes and A. Vairo, Phys. Rev. D 64, 076010 (2001); Phys. Lett. B 576, 314 (2003); A. Vairo, Nucl. Phys. Proc. Suppl. 133, 196 (2004).

[13] H. Witala, J. Golak, R. Skibinski, W. Glockle, W. N. Polyzou and H. Kamada, Phys. Rev. C 77, 034004 (2008)

[14] L. Girlanda, S. Pastore, R. Schiavilla, and M. Viviani, unpublished.

[15] S. Weinberg, The Quantum Theory of Fields (Cambridge University Press, 1995), vol. I. 